\title{
Lernschöpfungskette Forschung - von der Idee bis zur fertigen Abschlussarbeit
}

\section{Barbara Engler und Julia Gerstenberg}

Die Umsetzung des anspruchsvollen Konzepts „Forschendes Lernen“ gestaltet sich übersichtlicher und weniger schwierig, wenn es sowohl Studierenden als auch Lehrenden in Etappen nähergebracht und der akademische Nachwuchs mit dem Forschungsprozess in kleinen Einheiten vertraut gemacht wird. In diesem Beitrag werden die Vorteile einer Sequenzierung des Forschenden Lernens mit Fokus auf das Lernen des Forschens beleuchtet und anhand des Qualitätspakt-Lehre-Projekts „Humboldt reloaded - Wissenschaftspraxis von Anfang an" ${ }^{\text {"1 }}$ an der Universität Hohenheim beispielhaft dargestellt.

\section{$1 \quad$ Einleitung}

Die verschiedenen Definitionen des Begriffs „Forschendes Lernen“ sind hinsichtlich der Bestandteile, die Forschendes Lernen aufweisen muss, nicht einheitlich. Forschendes Lernen umfasst nach der Definition von Huber den gesamten Forschungsprozess: Die Lernenden finden selbstständig ihre Forschungsfrage, gestalten den gesamten Forschungsprozess aktiv mit und erzielen dabei Erkenntnisse, die für

1 Zur weiteren Beschreibung des Projekts „Humboldt reloaded“ siehe Blum, Gerstenberg \& Engler (2019) oder https://humboldt-reloaded.uni-hohenheim.de/ [09.08.2019]. 
Dritte relevant sind (Huber, 2009). Das ist wohl die voraussetzungsreichste Form Forschenden Lernens. In einer Definition in erweitertem Sinne als forschungsbezogene Lehre werden inhaltlich drei Subkategorien unterschieden, bei denen die Lehre a) Forschungsergebnisse, b) Forschungsmethoden oder c) den gesamten Forschungsprozess ins Zentrum stellt (Rueß, Gess \& Deicke, 2016). Dabei gibt es auch die Möglichkeit, dass Lernende nur in Teilen einen Forschungsprozess durchlaufen können. Dazu gehört beispielsweise die Aneignung neuen Fachwissens oder das Einüben aktueller Fachmethodik (Healey \& Jenkins, 2009). Forschendes Lernen kann aber auch erfordern, dass die Studierenden selbst forschen und dabei alle Phasen der Forschung durchlaufen, auch wenn der Forschungsprozess dabei nicht notwendigerweise anhand einer einzelnen Fragestellung komplett erarbeitet werden muss (Reinmann, 2015).

Huber selbst räumt ein, dass Forschendes Lernen an Universitäten und Hochschulen meist schwer umsetzbar ist (Huber, 2009). Die Restriktionen, denen sich Agierende bei der Einführung von Forschendem Lernen an Universitäten generell gegenüber sehen, können aus unserer Sicht sein: mangelnde Zeit und fehlende finanzielle Ausstattung; im Curriculum ist diese Art Studienleistung nicht vorgesehen oder nicht gewünscht; es handelt sich um sehr große Studierendengruppen; in der Universitätsleitung oder in Gremien gibt es keine Fürsprecher für Forschendes Lernen; es fehlt schlicht an einer Initialzündung.

Mit einer Kette von aufeinander aufbauenden Berührungspunkten zwischen Lehre und Forschung, die über den Studienverlauf verteilt sind, kann es trotzdem gelingen, Forschendes Lernen in die Lehre einzubinden, niedrigschwellig zu beginnen und es langsam zu seiner Idealform zu steigern. Auf diese Weise können Studierende mit der Zeit Forschungskompetenzen entwickeln und Lehrende Möglichkeiten ausloten, ihre Forschung mit der Lehre zu verbinden. Abgeleitet vom Begriff der Wertschöpfungskette wird als Sinnbild für diese Herangehensweise der in Hohenheim entstandene Begriff der Lernschöpfungskette im Forschenden Lernen verwendet.

\section{Der Forschungsprozess im Forschenden Lernen}

Forschung kann je nach Wissenschaftsdisziplin ganz verschieden definiert werden, daher variiert auch der Forschungsprozess im Detail stark (siehe Mieg in diesem Sammelband). Für die fachübergreifenden Überlegungen zu Forschendem Lernen schlägt Huber (2013) den Forschungsprozess in folgenden vereinfachten Etappen vor: 

a. Einführung
b. Finden einer Fragestellung
c. Erarbeiten von Informationen
d. Erwerb von Methodenkenntnissen
e. Entwickeln eines Forschungsdesigns
f. Durchführung einer forschenden Tätigkeit
g. Erarbeitung und Präsentation der Ergebnisse
h. Reflexion

Beim Forschenden Lernen kann nun, wie in einigen Definitionen beschrieben, entweder der gesamte Forschungsprozess durchlaufen werden oder Teile davon.

Ziel ist es stets, dass die Studierenden Forschungskompetenz erlangen (Tremp \& Hildbrandt, 2012). Mit Blick auf die Unterteilung der Zielrichtung Forschenden Lernens liegt in diesem Beitrag der Schwerpunkt darauf, dass Forschen gelernt wird, und weniger darauf, dass Lernen gelernt wird (siehe die Klassifizierung von Forschendem Lernen nach Rueß, Gess \& Deicke, 2016).

\section{Die Wertschöpfungskette}

Die Etappen des Forschungsprozesses bauen entweder aufeinander auf, finden in Zyklen statt (Wildt, 2009) oder wiederholen sich in einzelnen Teilen. Mit jeder Etappe findet ein Kompetenzzuwachs der Studierenden statt. Diese stufenförmige Kompetenzerweiterung kann auch mit dem Wertezuwachs einer Wertschöpfungskette verglichen werden, wobei der Prozess der Wertschöpfung in einem vollkommen anderen Zusammenhang, nämlich bei der Produktion von Gütern und Dienstleistungen in Industrie und Wirtschaft, stattfindet. Dort werden bei jeder Prozessstufe Ressourcen in Form von Kapital, Zeit, Energie und weiteren Faktorinputs verbraucht und zusätzliche Werte geschaffen (Porter, 1996). Die jeweiligen Prozessstufen ergeben sich dabei aus den vorhandenen Ressourcen und dem zu erstellenden Gut. Die Wertschöpfungskette bei der Erstellung eines Brotes zum Beispiel beginnt mit der Produktion von Weizen. Dieser wird nach der Ernte gemahlen. Dabei erfolgt ein Wertzuwachs des Weizens vom Korn zum Mehl. Das Mehl wird in der Bäckerei zu Brot verarbeitet; auch damit ist ein Wertzuwachs verbunden. Jede Prozessstufe hat Ressourcen in Form von Energie, Zeit und Arbeitsleistung verbraucht.

Neben der Wertschöpfungskette einer Produktion gibt es die Wertschöpfungskette einer kontinuierlichen Dienstleistung, die sich von Ersterer etwas unterscheidet. Dienstleistungen weisen vier Charakteristika auf: Immaterialität, zeitliches Zu- 
sammenfallen von Produktion und Konsum, Nichtlagerfähigkeit und Integration des externen Faktors (Kürble, 2006). Eine kontinuierliche Dienstleistung tritt beispielsweise bei der ärztlichen Behandlung von chronisch Kranken auf (siehe Beispiele Tab. 1).

\section{$4 \quad$ Die Lernschöpfungskette}

\subsection{Allgemein}

Die Charakteristika der Wertschöpfungskette einer kontinuierlichen Dienstleistung lassen sich auch auf die Lernschöpfungskette übertragen (Tab. 1). Sowohl bei der Bereitstellung der kontinuierlichen Dienstleistung als auch bei den einzelnen Stufen der Lernschöpfungskette werden Ressourcen in Form von Zeit, Kapital, Energie und Arbeitsleistung verbraucht und es wird dadurch ein Mehrwert - eine erweiterte Kompetenz - erzielt.

Aus dem Bereich der Didaktik und der Lernforschung ist bekannt, dass das Lernen neuer Inhalte an das vorhandene Wissen anknüpft (siehe z. B. Pellegrino, 2003). Je mehr Vorwissen vorhanden ist, desto höheren Nutzen können Lernende aus dem bereitgestellten Lernangebot ziehen (Kirschner, Sweller \& Clark, 2006). Mit jedem neu gelernten Inhalt, jeder neu erworbenen Fähigkeit steigt auch die Kompetenz der Studierenden. Es werden also in jeder Lerneinheit Werte - Überzeugungen, Wissen und Kompetenzen - erzeugt.

Auf das Forschende Lernen übertragen bedeutet das, dass die komplex ausgestaltete Forschungspraxis für die Lernenden in überschaubare, leistbare Einheiten mit Zwischenräumen zur Reflexion untergliedert werden kann. Dabei kann die Komplexität reduziert werden, indem entweder auf einzelne Teilaufgaben fokussiert oder die Forschungsfrage eingegrenzt wird (Tremp \& Hildbrand, 2012).

Werden Studierende in dieser gegliederten Form an Forschung herangeführt, können sie bereits in der Studieneingangsphase beginnen, ihre Forschungskompetenz aufzubauen (Tremp \& Hildbrand, 2012). Verschiedene Phasen des Forschens können in einzelnen Etappen geübt und gelernt werden (Reinmann, 2015). Ein niederschwelliger Einstieg in den Forschungsprozess kann durch Aufteilung komplexer Sachverhalte in kleine und einfach zu untersuchende Fragestellungen gelingen, beispielsweise in den Lebenswissenschaften (Selje-Aßmann et al., 2017). Diese Überlegungen gehen in die gleiche Richtung wie die Vorschläge von Tremp und Hildbrand: Sie legen dar, dass sich Forschendes Lernen innerhalb eines komplexen Forschungsthemas auf Teilaspekte der übergeordneten Forschungsfrage oder auf 
Tab. 1 Gegenüberstellung der Charakteristika einer Wertschöpfungskette einer kontinuierlichen Dienstleistung mit einer Lernschöpfungskette im Forschenden Lernen

\begin{tabular}{|c|c|c|}
\hline Charakteristika & $\begin{array}{l}\text { kontinuierliche } \\
\text { Dienstleistung }\end{array}$ & Forschendes Lernen \\
\hline Immaterialität & $\begin{array}{l}\text { Dienstleistungen sind nicht } \\
\text { materieller Natur und somit } \\
\text { mit den menschlichen Sinnen } \\
\text { nicht vor dem Kauf wahrzu- } \\
\text { nehmen, wie z. B. die Be- } \\
\text { handlung eines chronisch } \\
\text { Kranken durch einen Arzt. }\end{array}$ & $\begin{array}{l}\text { Auch der Forschungsprozess } \\
\text { ist immateriell. Wie sehr die } \\
\text { Studierenden von der Teil- } \\
\text { habe an der Forschung pro- } \\
\text { fitieren, lässt sich erst im } \\
\text { Nachhinein feststellen. }\end{array}$ \\
\hline $\begin{array}{l}\text { Zeitliches } \\
\text { Zusammenfallen } \\
\text { von Produktion } \\
\text { und Konsum }\end{array}$ & $\begin{array}{l}\text { Dienstleistungen werden in } \\
\text { dem Moment konsumiert, in } \\
\text { dem sie produziert werden. } \\
\text { Bsp.: Die medizinische } \\
\text { Untersuchung und die Be- } \\
\text { handlung finden gleichzeitig } \\
\text { statt. }\end{array}$ & $\begin{array}{l}\text { Forschendes Lernen findet in } \\
\text { dem Moment statt, in dem } \\
\text { am Forschungsobjekt gear- } \\
\text { beitet wird. }\end{array}$ \\
\hline $\begin{array}{l}\text { Nichtlager- } \\
\text { fähigkeit }\end{array}$ & $\begin{array}{l}\text { Wird eine Dienstleistung } \\
\text { nicht zu dem vereinbarten } \\
\text { Termin konsumiert, findet sie } \\
\text { nicht statt. Der Termin beim } \\
\text { Arzt verfällt. }\end{array}$ & $\begin{array}{l}\text { Forschung befindet sich in } \\
\text { einem ständig fortschreiten- } \\
\text { den Prozess. Wird an einem } \\
\text { bestimmten Zeitpunkt nicht } \\
\text { an der Forschung teilgenom- } \\
\text { men, so kann dieser spezifi- } \\
\text { sche Lernzuwachs nicht } \\
\text { mehr stattfinden. }\end{array}$ \\
\hline $\begin{array}{l}\text { Integration des } \\
\text { externen Faktors }\end{array}$ & $\begin{array}{l}\text { Externer Faktor: Patient. } \\
\text { Der Arzt kann seine Behand- } \\
\text { lung nur unter Einbeziehung } \\
\text { des Patienten zur Verfügung } \\
\text { stellen. }\end{array}$ & $\begin{array}{l}\text { Externer Faktor: Studierende. } \\
\text { Forschendes Lernen ist nur } \\
\text { durch Einbeziehung von Ler- } \\
\text { nenden möglich. }\end{array}$ \\
\hline
\end{tabular}

(eigene Darstellung basierend auf Kürble, 2006)

eine Teilaufgabe des Forschungsprozesses, wie beispielsweise wissenschaftliches Schreiben, statistische Auswertung von Daten oder Literaturrecherche, beziehen lässt (Tremp \& Hildbrand, 2012). 
Forschendem Lernen wird ein großes Potenzial zur Förderung der unterschiedlichen Kompetenzen zugeschrieben. Gess, Deicke und Wessels beschreiben in ihrer Arbeit eine Vielzahl dieser Kompetenzziele. Da sie es nicht für möglich halten, alle Kompetenzziele in einer Veranstaltung zu verfolgen, muss die Lehrperson unter diesen eine Auswahl treffen. Im Umkehrschluss bedeutet das, den Studierenden mehrere Formen von Forschendem Lernen im Studium zu ermöglichen. Damit haben die Studierenden die Wahl, welche Kompetenzen sie selbst ausbilden möchten (Gess, Deicke \& Wessels, 2017).

Es gibt verschiedene Modelle zur Erfassung der Kompetenzen, die durch Forschendes Lernen erzielt werden. Schneider und Wildt entwickelten ein Kompetenzstufenmodell mit vier Stufen und verbinden diese mit den Phasen eines idealtypischen Forschungszyklus (Schneider \& Wildt, 2007; Wildt, 2009). Auch das Research-Skill-Development Framework (RSD) (Willison \& O'Regan, 2007), das sich in sechs Kompetenzbereiche gliedert, orientiert diese am Ablauf des Forschungsprozesses und gibt fünf Level steigender studentischer Autonomie an. Beide Modelle verdeutlichen, dass jede einzelne durchlaufene Etappe im Forschungsprozess einen Kompetenzzuwachs aufseiten der Studierenden ermöglicht. Thiel und Böttcher wählen einen anderen Ansatz für ihr Kompetenzerfassungsmodell. Sie gliedern die fächerübergreifende Forschungskompetenz in ihrem RMKR-W-Modell, ungebunden an die Phasen des Forschungsprozesses, in Recherchekompetenz, Methodenkompetenz, Kommunikationskompetenz und Reflexionskompetenz, ergänzt um die fachliche Dimension des Wissens (Thiel \& Böttcher, 2014).

\subsection{Die Lernschöpfungskette in der Umsetzung}

An der Universität Hohenheim in Stuttgart können Bachelorstudierende unter anderem durch das seit 2011 (Laufzeit voraussichtlich bis 2020) durchgeführte Qualitätspakt-Lehre-Projekt „Humboldt reloaded - Wissenschaftspraxis von Anfang an" den Forschungsprozess im Ganzen sowie in Teilschritten vollziehen. Die Teilschritte werden im Folgenden beschrieben:

- Orientierung und Initiation ins Studium: Bereits vor Beginn des eigentlichen Studiums sind die zukünftigen Erstsemester eingeladen, die Fächervielfalt der Universität kennenzulernen. Die Lehrenden in der „Forschungsschnupperwoche" sind zugleich Forschende und führen die angehenden Studierenden in Kleingruppen in ihre spezifischen Fragestellungen ein. Die Fachbereiche werden von Lehrenden in ihren Arbeitsumgebungen und Forschungsfragen vorgestellt; so schauen die Erstsemester in Labore, auf Versuchshöfe oder auf Felder und 
erhalten einen ersten Einblick, wie Forschungsarbeit aussehen kann. In einem zweiten Schritt der Forschungsschnupperwoche teilen sich die Erstsemester in Kleingruppen auf und bearbeiten jeweils kleine fachspezifische Aufgaben. Die bisher durchgeführten Forschungsschnupperwochen der Fakultät Agrarwissenschaften fanden zum Thema Klimawandel statt. Studierende haben hier beispielsweise Keimversuche an einem Temperaturgradiententisch durchgeführt, um die Einflüsse des Klimas auf die Keimung zu simulieren. Für diese Projektarbeit, die dazugehörige Recherche und die Vorbereitung einer Präsentation haben die Studierenden zwei Tage Zeit. Lehrende stehen als Ansprechpersonen bereit. Über die Arbeit in der Kleingruppe lernen die Studierenden auch ihre Peers kennen. Zum Abschluss der Woche präsentieren die Kleingruppen ihre Arbeiten im großen Plenum und vor Lehrenden. Die freiwillige Teilnahme an der Forschungsschnupperwoche ist mit einem ECTS-Punkt in einem Wahlmodul im Bachelorstudium anrechenbar. Die Studierenden bekommen auf diese Weise einen Bezug zu der aktuellen Forschung und erfahren die Einheit von Forschung und Lehre. Sie erhalten durch die Forschungsschnupperwoche eine erste Orientierung für das Studium und können erste Kontakte zu Kommilitoninnen und Kommilitonen sowie Lehrenden knüpfen.

- Selbstreflexion und Karriereplanung: Das fakultative semesterbegleitende Seminar „Mit der eigenen Forschungsfrage motiviert durchs Studium“ wird für Studierende im ersten Bachelorstudienjahr angeboten und findet an vier Blockterminen statt. Ziel dieser Veranstaltung ist, dass die Studierenden sich anhand ihrer bisherigen Biografie ihrer Interessen, Fähigkeiten und Kenntnisse bewusst werden, für sie besonders interessante Themenfelder herausarbeiten und dann daraus eine persönliche Forschungsfrage erarbeiten, die sie durch das ganze Studium hinweg begleiten kann. Dazu sammeln die Studierenden beim ersten Termin Erfolgsgeschichten aus ihrem Leben, beschreiben einzelne genauer und arbeiten heraus, welche Fähigkeiten und Fertigkeiten sie brauchten, um jeweils das Ziel zu erreichen. Durch den Vergleich der Geschichten treten die persönlichen Stärken hervor. Um sich dieser noch einmal auf eine andere Weise bewusst zu werden, arbeiten sich die Studierenden dann durch einen Katalog von Tätigkeitsverben, unter denen sie immer wieder diejenigen priorisieren, deren Tätigkeit sie lieber mögen als die der anderen in dieser Auswahl. Am Ende der Aufgabe bleiben wieder die eigenen Interessen und Stärken übrig. Als Nächstes werden große Karten auf dem Boden ausgelegt, die jeweils einen Fachbereich der Hochschule darstellen und in Gänze alle Bereiche abdecken, sodass die Studierenden einen Überblick über die gesamte Fächervielfalt - auch über ihren Studiengang hinaus - erhalten. So entdecken die Studierenden neue interessante Fächer, zu denen sie bis zum nächsten Treffen eine Mindmap oder 
TagCloud mit den dazu recherchierten Inhalten und Forschungsfragen erstellen. Während des zweiten Treffens vertiefen die Studierenden Themen, die sie besonders interessant finden, indem sie im Brainstorming jeweils die möglichen Inhalte eines imaginären Magazins oder die Buchtitel einer imaginären Bibliothek zu ihren Themen sammeln und dann in der Gruppe vorstellen. Zum dritten Termin schreiben die Studierenden in drei sich überlappende Kreise jeweils ein Schlüsselwort zu den drei Themen ihres größten Interesses. Dann werden die Blätter mit den Schlüsselwörtern in den Schnittmengen in der Seminargruppe von Person zu Person gereicht und jeder schreibt seine Themen-Assoziationen dazu auf. Mit diesem zusätzlichen Input aus der Gruppe beginnen die Studierenden, ihre Forschungsfragen zu formulieren, die dann noch einmal in der Gruppe besprochen werden. Mit dem Hinweis, sich Unterstützerinnen und Unterstützer für die Bearbeitung der Forschungsfragen zu suchen, schließt das Seminar. Die individuelle Forschungsfrage ist Ausdruck für die persönliche innere Motivation. Diese klar herausgearbeitet zu haben, stärkt das Engagement für das eigene Studium und verleiht Durchhaltekraft und Lösungsorientierung auch in schwierigen Fächern (Hartung, 2018).

- Fachwissen und -theorien kennenlernen: In Vorlesungen, Seminaren und Übungen lernen Studierende die theoretischen Grundlagen ihres Faches. Hier werden ihre Fachkompetenzen gefördert. Während andere der aufgeführten Elemente der Lernschöpfungskette im Studium meist frei wählbar sind, ist dieses Element obligatorisch.

- Wissenschaftliche Methoden erlernen und üben: Es gibt verschiedene Angebote, in denen Aspekte wie wissenschaftliches Schreiben, statistische Datenerhebung und -auswertung oder die korrekte Darstellung wissenschaftlicher Ergebnisse erlernt und geübt werden können. Hiermit können die Studierenden je nach Bedarf fachübergreifende Methodenkompetenzen aufbauen und vertiefen.

- Begleitet den Forschungsprozess ganz oder teilweise durchlaufen: In Kleingruppen nehmen die Studierenden an aktuellen Forschungsvorhaben teil. Je nach Zeitaufwand, Forschungsgegenstand, Gruppengröße und Vorkenntnissen lernen sie dabei teilweise oder vollständig den Forschungsprozess in größerer oder kleinerer Gestaltungsfreiheit kennen. In den meisten Fällen ist die Fragestellung der studentischen Forschung vorgegeben und nicht frei wählbar. Das liegt daran, dass die betreuenden Personen Projekte mit Forschungsthemen entlang ihrer eigenen Forschungsfragen anbieten. Die Studierenden können aus den angebotenen Themen das für sie interessanteste auswählen. Die Inhalte der studentischen Forschungsprojekte sind so vielfältig wie die Forschung selbst. Auch die Bestandteile der studentischen Forschungsprojekte unterscheiden sich zum Teil beträchtlich: Wird in einem Projekt sehr viel Wert auf eine ausführliche 
Literaturrecherche gelegt, liegt in einem anderen Projekt das Hauptaugenmerk auf der statistischen Auswertung.

- Präsentation und Reflexion der eigenen Forschungsarbeit: Zur Ergebnissicherung und zur Abrundung der eigenen Erfahrung des Forschungsprozesses findet jedes Jahr eine studentische Tagung statt. Dazu schreiben die Teilnehmenden jedes studentischen Forschungsprojekts ein Abstract, das dann in einem Tagungsband veröffentlicht wird, und erstellen ein wissenschaftliches Poster. Während einer Posterausstellung präsentieren die Studierenden ihre Arbeiten und stehen für Rückfragen und Diskussionen bereit. Durch diese öffentliche Veranstaltung erfahren die Studierenden Selbstwirksamkeit und stärken ihre kommunikativen Kompetenzen.

- Gesamten Forschungsprozess durchlaufen: Das eigenständige Forschen und das Durchlaufen des gesamten Forschungsprozesses können im Rahmen einer Bachelor- und Masterarbeit erfolgen oder auch in Forschungsprojekten, die von den Studierenden selbst initiiert werden. Die Evaluation von „Humboldt reloaded“ erwies, dass viele Studierende sich nach der Teilnahme an einem studentischen Forschungsprojekt im Studium weiter mit dem Thema ihres Projekts befassen - als Hilfskraft am Fachgebiet, welches das Projekt anbot, in einem Seminar oder auch in der Bachelorarbeit (Oberhauser et al., 2014). Das Verfassen einer Abschlussarbeit ist für alle Studierenden eine Pflichtaufgabe, ob nun Schritte des Forschenden Lernens vorangingen oder nicht. Es ist jedoch davon auszugehen, dass die Selbstständigkeit und die forschungsmethodische Kompetenz höher sind als ohne Vorerfahrungen und Vorkenntnisse. Mitunter haben die Etappen vorher zu Kontakten innerhalb von Fachbereichen geführt und die Studierenden haben Ansprech- und Betreuungspersonen für ihr wissenschaftliches Arbeiten gefunden.

Die im Folgenden dargestellte Lernschöpfungskette im Forschenden Lernen zeigt, wie sich durch die Aneinanderreihung der einzelnen Teilaspekte des Forschenden Lernens der Kompetenzzuwachs verändert. Jeder einzelne Teilschritt hat seine Berechtigung und ermöglicht es den Studierenden, eine umfassende Forschungskompetenz systematisch aufzubauen. Dabei besteht die Möglichkeit, die Teilnahme an einzelnen Stufen zu wiederholen. Zwischen den Etappen der Lernschöpfungskette besteht für die Studierenden die Möglichkeit, das Erlernte zu reflektieren. 


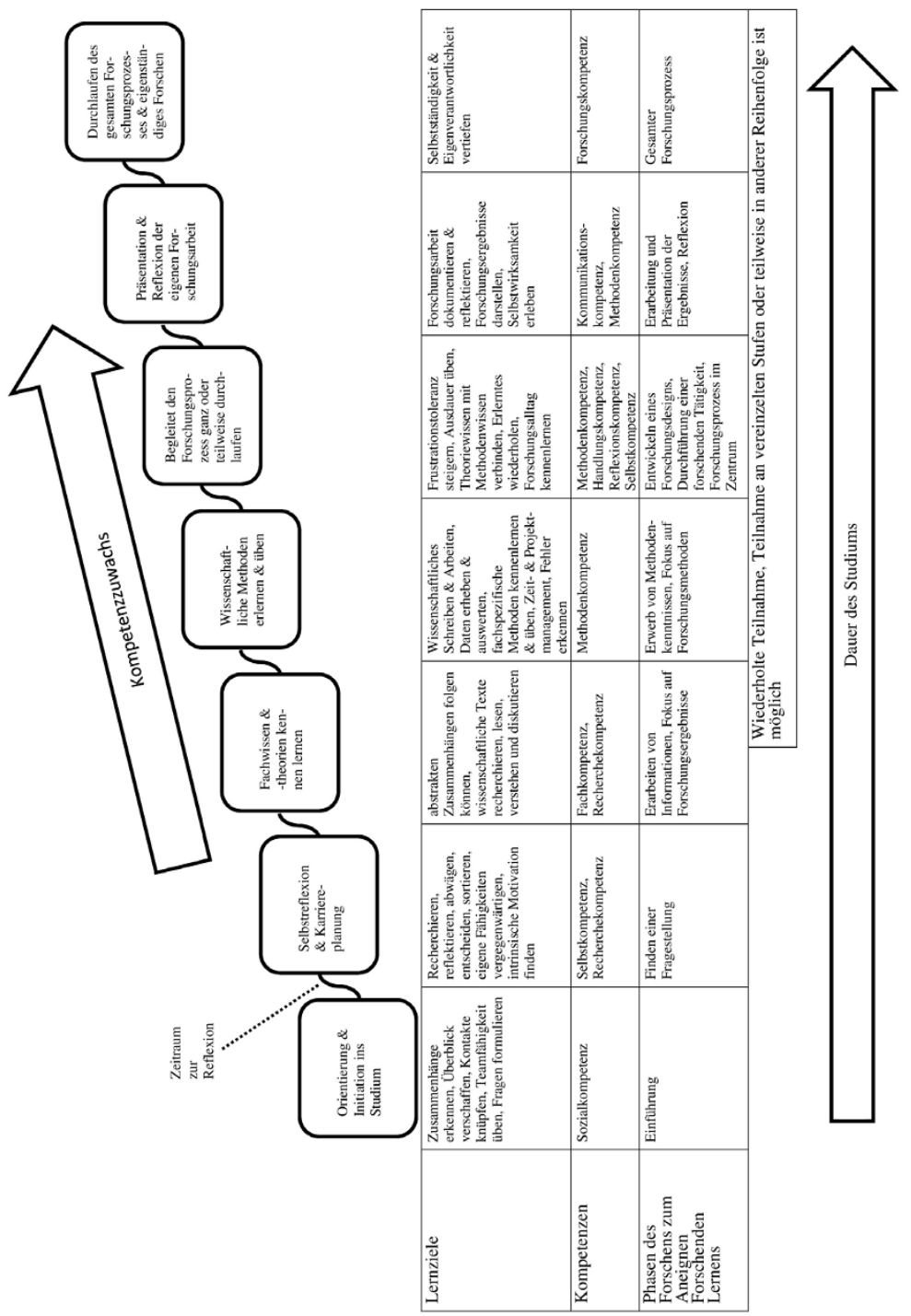

Abb. 1 Schematische Darstellung der Lernschöpfungskette im Forschenden Lernen, mit Lernzielen und Kompetenzen, angelehnt an einen idealtypischen Forschungsprozess

Quelle: eigene Abbildung, angelehnt an Huber (2013), Schneider und Wildt (2007), Rueß, Gess \& Deicke (2016), Thiel und Böttcher (2014) und Willison und O’Regan (2007) 


\section{$5 \quad$ Vorteile und Bedingungen einer Sequenzierung des Forschenden Lernens in einer Lernschöpfungskette}

Anhand der studentischen Rückmeldungen und des Engagements Studierender, die an Elementen der Lernschöpfungskette - vor allem Forschungsschnupperwoche, Orientierungskurs und studentischen Forschungsprojekten - teilnahmen, ist zu vermuten, dass die Lernschöpfungskette positive Effekte für die Lernenden zeigt. Eine Sequenzierung des Forschenden Lernens ermöglicht es Studierenden durch die Pausen zwischen den verschiedenen Angeboten, das bereits Erlernte zu verarbeiten. Je nach Ausgestaltung der Formate kann es Studierenden auch zuerst ermöglicht werden, einen Forschungsprozess zu beobachten, die Vorgehensweise kennenzulernen und dann erst selbst aktiv in die Forschung einzusteigen. Das Forschende Lernen im engeren Sinne, also das komplette Durchlaufen des Forschungsprozesses, ist für die Studierenden sehr voraussetzungsreich. Eine Sequenzierung kann einer möglichen Überforderung vorbeugen. Da die Studierenden wiederholt an Elementen des Forschendes Lernens teilnehmen können, eröffnet sich die Möglichkeit, diese Projekte in ihrer Komplexität zu steigern, sie also in Form eines "Spiralcurriculums“ zu durchlaufen (Bruner, 1970; siehe auch Huber in diesem Sammelband).

Für die Lehrenden ergibt die Lernschöpfungskette eine Verteilung der zu lernenden Forschungskompetenzen auf einen längeren Zeitraum und auf die Schultern vieler Lehrender. So muss nicht in einem studentischen Forschungsprojekt bei den Anfängen des wissenschaftlichen Arbeitens und des Forschenden Lernens begonnen werden. Wenn es vorher niederschwellige Angebote gibt, kann ein bestimmtes Niveau vorausgesetzt werden. Lehrende können Studierende zu anderen Teilschritten Forschenden Lernens verweisen, da sie bei einer bestehenden Lernschöpfungskette einen Überblick haben, welche anderen Angebote es gibt. Für Lehrende, die im Bereich des Forschenden Lernens erste Angebote ausprobieren möchten, eröffnen sich durch die Möglichkeit der Untergliederung ganz andere Lehrformate. Sie sind nicht aufgefordert, mit den Studierenden den kompletten Forschungszyklus zu bearbeiten, sondern können sich in Teilaspekten ausprobieren. Dies vereinfacht den Lehrenden den Einstieg in das Forschende Lernen. Aufseiten der Lernenden ergibt sich noch ein weiterer Vorteil der Lernschöpfungskette im Forschenden Lernen: Je nach Verfügbarkeit und eigenem Tempo können die Angebote von den Lernenden bedarfsgerecht genutzt werden. Das Zürcher Modell führt dies konsequent zu Ende: Von den Studierenden werden forschungsorientierte Leistungsnachweise erbracht; diese Leistungsnachweise dokumentieren die erfolgreiche Bewältigung einzelner Etappen des Forschungsprozesses (Tremp \& Hildbrand, 2012). Es bedarf der Anleitung oder Begleitung der Studierenden, da sie sonst den Weg der aufeinander aufbauenden Elemente möglicherweise nicht erkennen und 
die Angebote dazu nicht nutzen. Hier ist eine Moderation der Hochschule oder der Studiengangsleitung hilfreich.

\section{Strukturelle Verankerung der Lernschöpfungskette}

Die einzelnen Glieder der Lernschöpfungskette müssen miteinander verbunden sein, damit sie als eine Kette erkannt werden können. Das bedeutet für die Sequenzierung des Forschenden Lernens, dass eine gute Absprache der Lehrenden untereinander und/oder ein struktureller Rahmen im Studiengang oder in der Studienberatung, auf den immer wieder Bezug genommen werden kann, notwendig ist. So können unterschiedliche Auffassungen der Lehrenden zu Forschung und Wissenschaft, verschiedene Prioritäten bezüglich Inhalten, Methoden und Darstellungen für das Lernen des Forschens überbrückt werden. Die individuellen Herangehensweisen der Lehrkräfte können für die Studierenden verwirrend sein. Daher ist es wichtig, sie zwischen den einzelnen Sequenzen der Lernschöpfungskette reflektieren zu lassen, was sie gelernt, wie weit sie einen Forschungsprozess kennengelernt haben, und ihre Perspektive vom Teilbereich auf den ganzen Prozess wechseln zu lassen. Die Verständigung über die Moderation dieses gesamten Lernprozesses unter den Lehrenden kann dabei helfen, für das Anleiten zu Forschendem Lernen auf eine gemeinsame Grundhaltung zu kommen.

An der Universität Hohenheim findet Forschendes Lernen in verschiedenen Formaten statt. Einige davon sind freiwillig und trotzdem in Wahl- oder Pflichtmodulen anrechenbar. Weitere Formate sind Pflichtleistungen in den Studiengängen. Wenn das Konzept der Lernschöpfungskette konsequent umgesetzt werden soll, müssen alle Angebote des Forschenden Lernens in die verschiedenen Kettenglieder einsortiert und aufeinander abgestimmt werden.

Soll Forschendes Lernen ein Profilmerkmal der Hochschule sein, können grundlegende Forschungskompetenzen innerhalb verpflichtender Studienleistungen vermittelt werden. Es besteht dann die Möglichkeit, dass die Studierenden durch darauf aufbauende, frei zu wählende Angebote ihre Forschungskompetenzen weiterentwickeln. 


\section{$7 \quad$ Fazit}

Das hier beschriebene Konzept stellt dar, dass Prozesse in der Industrie, in Form der Wertschöpfungskette, und in der universitären Lehre, insbesondere im Forschenden Lernen in Form einer Lernschöpfungskette, Parallelen aufweisen. In beiden Bereichen findet ein Wert- bzw. Kompetenzzuwachs in Stufen statt. Eine Unterteilung des Forschungsprozesses in Teilschritte ist für die Studierenden ebenfalls in jeder Etappe mit einem Zuwachs an Wissen und Fähigkeiten verbunden. Studierende können auch von Teilprozessen des Forschenden Lernens profitieren. Lehrende, die sich in diesem Feld neu ausprobieren möchten, können sich durch eine Sequenzierung des Forschungsprozesses auf eine bestimmte Phase konzentrieren und die Studierenden an dieser Stelle gezielt begleiten.

Forschendes Lernen nach der Definition von Huber ist, wie eingangs erwähnt, sehr voraussetzungsreich (Huber, 2009). So wird viel Zeit zur Durchführung benötugt und die Studierenden müssen bereits in der Lage sein, den komplexen Forschungsprozess zu überblicken. Sind die Voraussetzungen nicht gegeben, sind aus unserer Sicht die Vorteile der Sequenzierung und der Wiederholung einzelner Schritte im Forschungsprozess für die Herausbildung der Forschungskompetenz der Studierenden gleich sinnvoll.

Mit dem Konzept der Lernschöpfungskette wird Forschendes Lernen im engeren Sinne - insbesondere dann, wenn nicht alle Teile feste Bestandteile des Studiums sind - nur zum Teil erreicht (Huber, 2014). Näher kann man dem Ideal kommen, sofern alle genannten Teile im Studienverlauf in einer Art Forschungs-Curriculum verpflichtend zu leisten sind, jedoch Freiheit bleibt, zu wählen - zum Beispiel, welches Thema in welchem Umfang wann im Studium bearbeitet werden soll. Dieser Ansatz kann unter der weiteren Definition des forschungsnahen Lehrens und Lernens verortet werden. Die dem Forschenden Lernen zugrunde liegende Intention der Vermittlung von Forschungskompetenz bleibt auch in der sequenzierten Form erhalten. Die Sequenzierung bietet immer wieder Anreize, sich dem Forschenden Lernen zu nähern und sich darin zu vertiefen. Letztendlich sind jedoch wieder die „Überzeugungstäter“, zur Verfügung stehendes Personal, sowie räumliche und materielle Ressourcen entscheidend. Auch die Absprachen der Lehrenden untereinander und ein gemeinsamer Austausch über die Entwicklungen in der Lernschöpfungskette sind wichtig. Ohne Begleitung und aktive Verbindung der Einzelelemente vermag sich der Reiz der sequenzierten Vermittlung Forschenden Lernens nicht zu entfalten. 


\section{Literatur}

Blum, M., Gerstenberg, J. \& Engler, B. (2019). Humboldt reloaded: Forschendes Lehren und Lernen im Grundstudium. In S. Kauffeld \& J. Othmer (Hrsg.), Innovative Lehre (S. 151-164). Heidelberg: Springer.

Bruner, J. S. (1970). Der Prozeß der Erziehung [The Process of Education]. Berlin: Berlin Verlag. Gess, C., Deicke, W. \& Wessels, I. (2017). Kompetenzentwicklung durch Forschendes Lernen. In H. A. Mieg \& J. Lehmann (Hrsg.), Forschendes Lernen: Wie die Lehre in Universität und Fachhochschule erneuert werden kann (S.79-90). Frankfurt: Campus.

Hartung, K. (2018). Die eigene Forschungsfrage finden. In H. A. Mieg \& J. Lehmann (Hrsg.), Forschendes Lernen: Ein Praxisbuch (S. 50-65). Potsdam: Verlag der Fachhochschule Potsdam.

Healey, M. \& Jenkins, A. (2009). Developing Undergraduate Research an Inquiry. Verfügbar unter https://www.heacademy.ac.uk/knowledge-hub/developing-undergraduate-research-and-inquiry [20.10.2017].

Huber, L. (Hrsg.) (2009). Forschendes Lernen im Studium: Aktuelle Konzepte und Erfahrungen. Bielefeld: UVW.

Huber, L. (2013). Methodische Anregungen für den Umgang mit pragmatischen Schwierigkeiten im Forschenden Lernen. In L. Huber, M. Kröger \& H. Schelhowe (Hrsg.), Forschendes Lernen als Profilmerkmal einer Universität (S.247-255). Bielefeld: UVW.

Huber, L. (2014). Forschungsbasiertes, Forschungsorientiertes, Forschendes Lernern: alles dasselbe? Ein Plädoyer für die Verständigung über Begriffe und Unterscheidungen im Feld forschungsnahen Lehrens und Lernens. Das Hochschulwesen, 62(1 \& 2), S.32-39.

Kirschner, P., Sweller, J. \& Clark, R. (2006). Why Minimal Guidance During Instruction Does Not Work: An Analysis of Failure of Constructivist, Discovery, Problem-Based, Experiential, and Inquiry-Based Teaching. Educational Psychologist, 41(2), S. 75-86.

Kürble, P. (2006). Die unternehmensinterne Wertschöpfungskette bei Dienstleistungen am Beispiel der TV-Programmveranstalter (Arbeitspapier Nr. 4. FOM - Fachhochschule für Oekonomie \& Management). Essen.

Oberhauser, E., Schröter, D., Badermann, M. \& Kaschube, J. (2014). Wie wirkt Humboldt reloaded? Eine Untersuchung der Wirksamkeit der Humboldt reloaded - Projekte an der Universität Hohenheim. Abschlussbericht. Verfügbar unter https://humboldt-reloaded. uni-hohenheim.de/evaluation [22.11.2017].

Pellegrino, J. W. (2003). Connecting Learning Theory and Instruction: Principles, Practices, and Possibilities. In F. Achtenhagen \& E. G. John (Hrsg.), Die Lehr-Lern-Perspektive: The Teaching-Learning Perspective (S. 17-42). Bielefeld: Bertelsmann.

Porter, M. E. (1996). Wettbewerbsvorteile: Spitzenleistungen erreichen und behaupten. Frankfurt: Campus.

Reinmann, G. (2015). Heterogenität und forschendes Lernen: Hochschuldidaktische Möglichkeiten und Grenzen. In B. Klages, M. Bonillo, S. Reinders \& A. Bohmeyer (Hrsg.), Gestaltungsraum Hochschullehre: Potenziale nicht-traditionell Studierender nutzen (S. 121-137). Opladen: Budrich UniPress.

Rueß, J., Gess, C. \& Deicke, W. (2016). Forschendes Lernen und forschungsbezogene Lehre - empirisch gestützte Systematisierung des Forschungsbezugs hochschulischer Lehre. Zeitschrift für Hochschulentwicklung, 11(2), S.23-44. 
Schneider, R. \& Wildt, J. (2007). Forschendes Lernen in Praxisstudien: Ein hochschuldidaktisches Konzept zur Förderung professioneller Kompetenzen in der Lehrerbildung. Journal für Hochschuldidaktik, 18(2), S. 11-15.

Selje-Aßmann, N., Poll, C., Tisler, M., Gerstenberg, J., Blum, M. \& Fleischer, J. (2017). Forschendes Lernen in den Lebenswissenschaften. In H. A. Mieg \& J. Lehmann (Hrsg.), Forschendes Lernen: Wie die Lehre in Universität und Fachhochschule erneuert werden kann (S. 200-210). Frankfurt: Campus.

Thiel, F. \& Böttcher, F. (2014). Modellierung fächerübergreifender Forschungskompetenzen: Das RMKR-W-Modell als Grundlage der Planung und Evaluation von Formaten forschungsorientierter Lehre (I 2.10, S. 109-124). In B. Berendt, H. P. Voss \& J. Wildt (Hrsg.), Neues Handbuch Hochschullehre: Lehren und Lernen effizient gestalten. Berlin: Raabe.

Tremp, P. \& Hildbrand, T. (2012). Forschungsorientiertes Studium - universitäre Lehre: Das „Zürcher Framework“ zur Verknüpfung von Lehre und Forschung. In T. Brinker \& P. Tremp (Hrsg.), Einführung in die Studiengangentwicklung (Blickpunkt Hochschuldidaktik 122) (S. 101-116). Bielefeld: Bertelsmann.

Wildt, J. (2009). Forschendes Lernen: Lernen im „Format“ der Forschung. Journal für Hochschuldidaktik, 20(2), S.4-7.

Willison, J. \& O’Regan, K. (2007): Commonly known, commonly not known, totally unknown: a framework for students becoming researchers. Higher Education Research \& Development. 26(4), S. 393-409.

\section{Danksagung}

Unser Dank geht an Dr. Natascha Selje-Aßmann für die kritische Durchsicht des Manuskripts und die vielen hilfreichen Kommentare.

\section{Zu den Autorinnen}

Julia Gerstenberg, M.A., war Wissenschaftliche Mitarbeiterin an der Universität Hohenheim, wo sie das Projekt „Humboldt reloaded - Wissenschaftspraxis von Anfang an" inhaltlich koordinierte. Mit ihrem Hintergrund als Kulturwissenschaftlerin und Mediatorin war es ihre Hauptaufgabe, die verschiedenen Beteiligtengruppen - Studierende, wissenschaftliche Mitarbeiter, Professoren aus verschiedenen Fachbereichen - zur Organisation verschiedener Formen Forschenden Lernens zusammenzubringen und die fachübergreifende Arbeit zu moderieren. E-Mail: julia.gerstenberg@hs-mittweida.de

Dr. Barbara Engler ist Diplom-Agraringenieurin und war an der Fakultät Agrarwissenschaften Koordinatorin für das Projekt „Humboldt reloaded“. Hierbei war ihre Aufgabe unter anderem, die Etablierung und Verankerung des Forschenden Lehrens und Lernens in der Fakultät Agrarwissenschaften zu begleiten. E.Mail: b.engler@uni-hohenheim.de 
Open Access Dieses Kapitel wird unter der Creative Commons Namensnennung 4.0 International Lizenz (http://creativecommons.org/licenses/by/4.0/deed.de) veröffentlicht, welche die Nutzung, Vervielfältigung, Bearbeitung, Verbreitung und Wiedergabe in jeglichem Medium und Format erlaubt, sofern Sie den/die ursprünglichen Autor(en) und die Quelle ordnungsgemäß nennen, einen Link zur Creative Commons Lizenz beifügen und angeben, ob Änderungen vorgenommen wurden.

Die in diesem Kapitel enthaltenen Bilder und sonstiges Drittmaterial unterliegen ebenfalls der genannten Creative Commons Lizenz, sofern sich aus der Abbildungslegende nichts anderes ergibt. Sofern das betreffende Material nicht unter der genannten Creative Commons Lizenz steht und die betreffende Handlung nicht nach gesetzlichen Vorschriften erlaubt ist, ist für die oben aufgeführten Weiterverwendungen des Materials die Einwilligung des jeweiligen Rechteinhabers einzuholen.

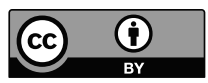

